\title{
Spray-on Ferroelectrics for Fabrication of Custom Tailored Composite Transducers for NDE and SHM
}

\author{
K. Sinding, A. Orr, B. Reinhardt L. Tien, N. Malarich and B. Tittmann \\ Department of Eng. Science \& Mechanics \\ Pennsylvania State University \\ University Park, PA
}

Applications for non-destructive evaluation (NDE) and structural health monitoring (SHM) in principle can be performed at various temperatures provided the transducer can reliably operate under those conditions. For are high temperature materials such as Lithium Niobate that can be used for NDE a coupling medium is required to aid in wave propagation between the transducer and system of interest. Traditional methods require either pressure contacts or a high temperature bonding medium, which can deteriorate over time. The sol-gel spray-on technology eliminates the need for this coupling medium and allows for an actuator that can be custom tailored to the specific application of interest. As nuclear reactors reach the end of their projected lifetime it is critical that the systems within the containment structure be continuously monitored. This spray-on technology allows for efficient on-line monitoring of a system at any operating temperature providing the proper transducer selection.

The current objective is to develop a class of spray-on ferroelectrics that do not require a bonding medium and enable custom tailoring of composite transducers adapted for different combinations of efficiency and temperature. For example, if a high-temperature application is anticipated, the composition of the sol-gel can involve constituents that favor ultrasonic properties, while the powder could involve constituents that still function ultrasonically at high temperature.

This paper presents details of the deposition method without requiring a bonding medium. It also shows results on the effects of varying the weight percent of the composite Lead Zirconate Titanate/ Bismuth titanate transducers on the Curie temperature and efficiency of the composite Piezoelectrics. Results for several composites are given with $d_{33}$ values ranging from 20 to $60 \times 10^{-12}$ Coulomb/Newton along with temperature dependencies of the signal amplitude in pulse-echo experiments. These composite ferroelectrics are modeled using a micromechanics analysis code based on the "generalized method of cells" program.

Composite, Spray-on, Ferroelectric, BiTi/PZT, High Temperature

\section{INTRODUCTION}

Spray on transducers have attracted a significant amount of attention from a variety of different industries for applications ranging from monitoring in-ground gas pipes to structural health monitoring of heat exchangers in a nuclear power plants[1]. These applications vary widely in operation temperatures and the strongest signal possible is desired for each application. This combination of desired properties is difficult to find in one material. Thus a combination of two materials, bismuth titanate (BiTi) and lead zirconate titanate (PZT) can be combined in varying weight percent to vary the properties of the transducer for specific applications. PZT is a common ferroelectric that has a very high piezoelectric coefficient and a relatively low Curie temperature. BiTi on the other hand has a relatively high Curie temperature and a much lower piezoelectric coefficient [2].

It is also important to be able to model these composites in order to provide a basis to compare the experimental results to. The Micromechanics Analysis Code by Generalized Method of Cells program provided by NASA has been utilized to accurately model the composites at room temperature.

\section{METHODS}

To create a strong coupling between the transducer and the pipe, a spray-on method [3] has been adopted following the early work of Kobayashi in 2004. This method involves two constituents, a sol-gel solution and a powder. The solgel/powder composite consists of BiTi sol gel and varying weight percent of BiTi on PZT powders, the properties of each material can be found in Table 1 . The weight percent of each transducer has been varied from $20 \%$ BiTi to $100 \%$. Note that all weight percent expressed in this paper in both figures and text denote the weight percent of BiTi. The remaining weight percent is PZT. In the present procedure the substrate is covered with a mask to ensure only one surface is coated and the slurry is sprayed to create a smooth and even coating across the circumference. After each spray, the sample is placed on the hotplate at $400^{\circ} \mathrm{C}$ to pyrolize the ceramic. This process is repeated five times to create a thin $100 \mathrm{um}$, ceramic film on the substrate. The sample is then sintered using a crude blowtorch sintering process outline by Searfass [2]. After sintering, samples are electroded with sputtered platinum then Ag conductive paint which is fired onto the ceramic at $400{ }^{\circ} \mathrm{C}$. To pole the samples, they are lowered into silicon oil with a high voltage connected to the electrode and the pipe is grounded. Typical poling conditions are approximately $50 \mathrm{kV} / \mathrm{cm}$ and the oil is heated to $150{ }^{\circ} \mathrm{C}$. The sample will be held at temperature for 20 minutes with the applied field. Once the sample has been poled it is removed from the oil bath and air-cooled with the field still applied.

Table 1: Curie temperatures and efficiencies of materials used for composite transducers.

\begin{tabular}{|l|l|l|}
\hline Material & $\mathrm{T}_{\mathrm{c}}\left({ }^{\circ} \mathrm{C}\right)$ & $\mathrm{d}_{33(\mathrm{pC} / \mathrm{N})}$ \\
\hline $\mathrm{Bi}_{4} \mathrm{Ti}_{3} \mathrm{O}_{12}$ & $685[2]$ & $14[2]$ \\
\hline $\mathrm{Pb}\left(\mathrm{Zr}_{\mathrm{x}}, \mathrm{Ti}_{(1-\mathrm{x})}\right) \mathrm{O}_{3: \mathrm{X}=.48}$ & $385[4]$ & $585[4]$ \\
\hline
\end{tabular}




\section{THEORETICAL MODELING}

The theoretical modeling completed in this text utilizes the Micromechanics Analysis Code by Generalized Method of Cells 4.0 (MAC/GMC). This code was developed at NASA Glenn Research Center specifically to be used for the modeling of laminates and composite materials [5].

MAC/GMC employs a repeating unit cell (RUC) approach to accurately control the placement and quantities of the composites constituent materials. The RUC is the central building block for electro-mechanical modeling of the composites and is triply periodic. The RUC is assumed to extend infinitely in all three Cartesian directions, which for a thin film is not accurate; however, the method still can be used to model resultant material properties.

In Fig. 1 the matrix is represented by the white subcells, while the shaded sub-cell is the inclusion or fiber. For the modeling completed in this text the matrix material is BiTi $[5]$.

The generalized method of cells is governed by a set of differential equations that are constrained by a set of continuity conditions and a set of boundary conditions. The three governing differential equations are given as:

$$
\begin{gathered}
\epsilon_{i j j}=\frac{1}{2}\left(u_{i, j}+u_{j, i}\right) \\
\sigma_{i j, j}+b_{i}=0 \\
D_{i, j}=q_{b}
\end{gathered}
$$

The continuity conditions require that the boundaries of each sub-cell satisfy four basic laws for electro mechanical interactions:

1. Continuity of displacements at the interfaces between the sub cells

2. Continuity of tractions at the interfaces between the sub cells

3. Continuity of electric potentials at the interfaces between the sub cells

4. Continuity of normal electric displacements at the interfaces between the sub cells

Finally a set of boundary conditions can be applied to solve Eqns. 1-3. These Boundary

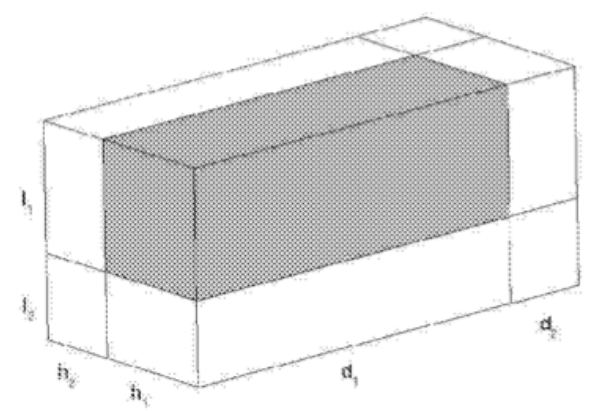

Figure 1: Triply Periodic RUC for electromechanical modelling

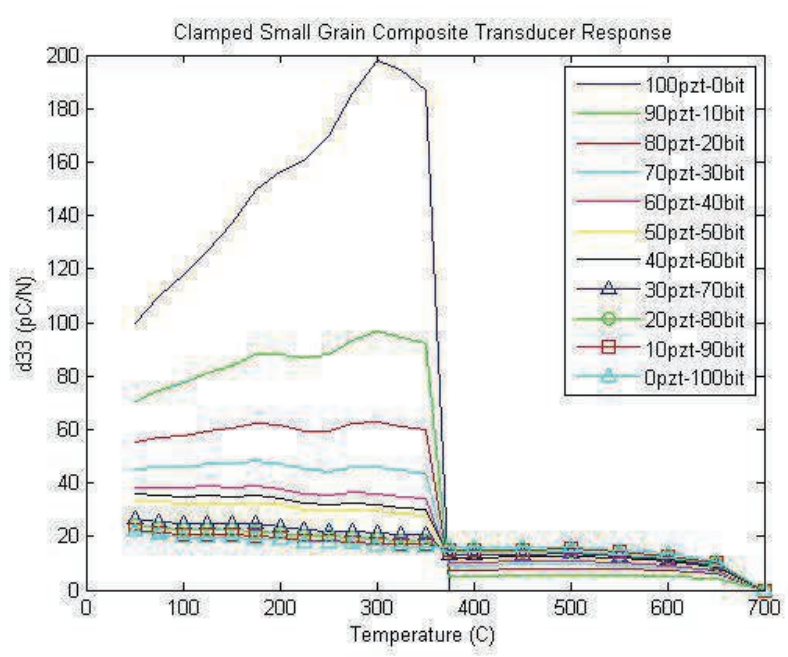

Figure 2: Composite transducer $\mathrm{d}_{33}$ as a function of temperature with clamping and grain size considerations.

Conditions are that the tractions, displacements, and electric displacements must have the same values at the left and right sides of the RUC, and the top and bottom of the RUC must also have the same values, which may be different from the sides of the RUC. To determine the macro-scale properties of the composite a volume averaging technique can be used.

To create an accurate model two additional effects must be considered. Since the ceramic is attached to the substrate the transducer is considered clamped since it cannot contract or expand in the 31 mode. The effective $d_{33}$ of a clamped transducer can be expressed as

$$
\left.\mathrm{d}_{33 \text { (eff) }}=\mathrm{d}_{33}+2 \mathrm{~d}_{31}\left[(-\mathrm{v} / \mathrm{c})-\mathrm{s}^{\mathrm{E}}{ }_{13}\right)\right] /\left(\mathrm{s}^{\mathrm{E}}{ }_{11+} \mathrm{s}^{\mathrm{E}}{ }_{12}\right)
$$

Torah then made the necessary measurements to reduce the equation for PZT to

$$
\mathrm{d}_{33 \text { (eff) }}=\mathrm{d}_{33}+1.33 \mathrm{~d}_{31}
$$

When the effective $d_{33}$ is calculated the result is $56 \%$ reduction in the effective $d_{33}$ of the free PZT transducer. The effective $d_{33}$ for BiTi is not effected by clamping[6]. Also, grain size must be considered. The unique processing conditions also must be considered in order to accurately model the piezoelectric response. These processing conditions result in much smaller grain sizes than an ideal case. Randall et.al. published the effects of grain size on $d_{33}$ [7]. After making these considerations the $d_{33}$ can be plotted as a function of temperature for a variety of compositions.

\section{RESULTS AND DISCUSSION}

Since these ferroelectric materials are intended to be used as transducers in high temperature applications the main focus of this section deals with the signals produced by the resulting 


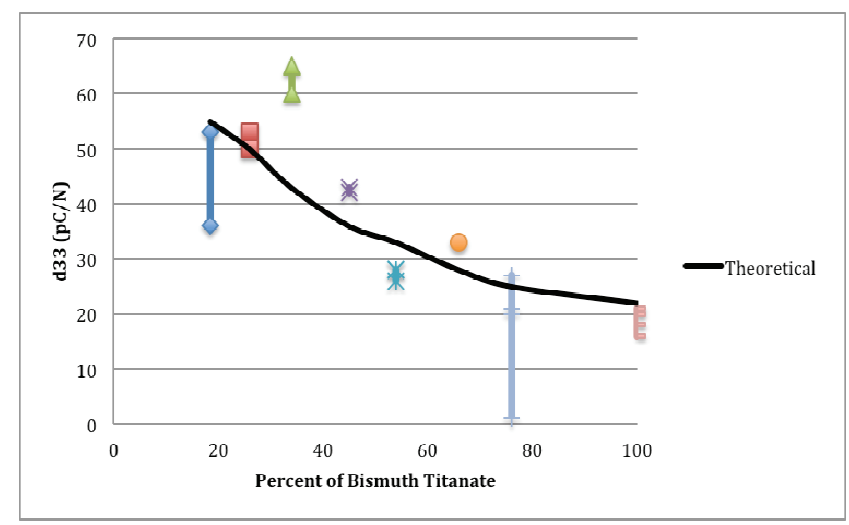

Figure 3: The effect if composition of a PZT-Bismuth Titanate transducer on the resultant $d_{33}$.

transducers. In addition, other properties such as the relative permittivity and density are also discussed.

The theoretical curve presented in Fig. 3 is a result of the theoretical modeling completed in Section three of this text. These predicted values consider clamping, grain size, and porosity. The experimental results follow the same trend as the theoretical values. The $r$ squared value of this set of data is 0.628 . There are a few outliers that reside within the data most notably the $\mathrm{d}_{33}$ value of 1 for $78 \%$ BiTi and $22 \%$ PZT. If this data point and other outliers are excluded, the $r$ squared value increases to 0.716 . This $r$ squared value while not ideal indicates that the proper material property considerations have been taken in theoretically modeling the sol-gel spray-on system.

An important property of a transducer is the center frequency and band width of the resulting signal. Fig. 4 demonstrates these properties. All of the samples were tested using a transmitter/receiver. This type of transmitter emits a single tone burst to the transducer. There is not a strong dependency of the transducer composition on the center frequency of the resulting transducer since the center frequency of a longitudinal wave is determined by the thickness of the transducer. The band width of the transducer is determined by the amount of damping, the pulse width, and the variation in thickness of the transducer [8]. Since the wave speed for BiTi and PZT are similar the center frequency of each transducer is highly dependent on the thickness of the transducer rather than on the composition of the composite. The bandwidth of the composite

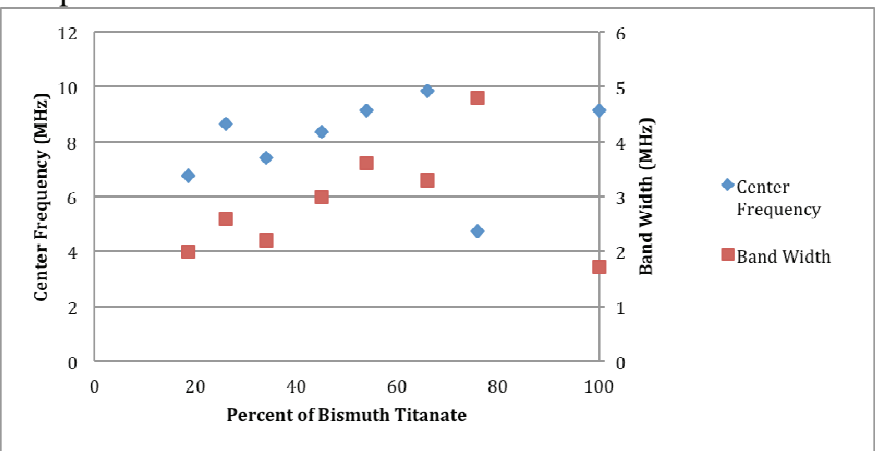

Figure 4: Center frequency and band width of composite transducers

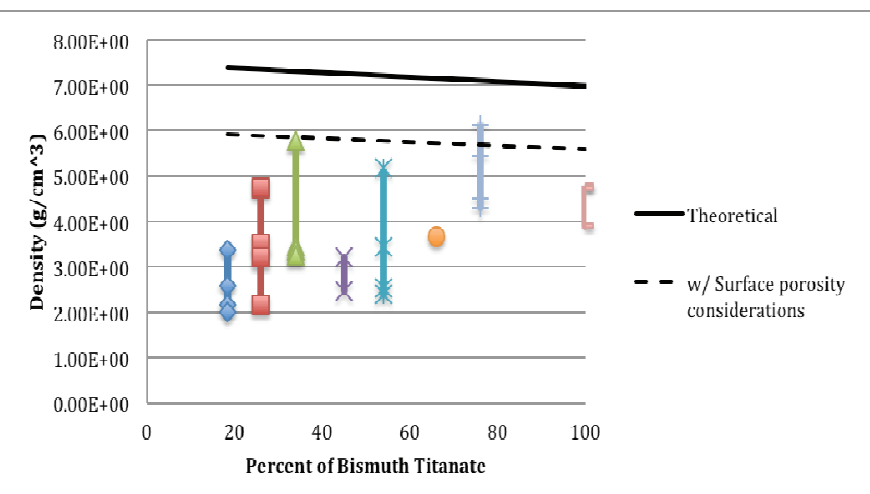

Figure 5: Density of composite transducers as a function of the composistion of the transducer. A theoretical density along with the surface approximated density have been included.

transducers are also not a strong function of the composition, but rather are determined by the testing conditions. These statements confirm the results presented in Fig. 4 that there is no observable trend in bandwidth or center frequency as a function of transducer composition.

The density of the composites is significantly lower than the theoretical density of the composites. The densities range from $26 \%$ to $86 \%$ of the theoretical density. A SEM image of the surface of the sample was taken to assess the porosity of the sample.

Relative permittivity and resistivity are also much lower than expected. These values have a strong dependence on the microstructure and density of the material. The anomalously low density of the spray on ferroelectrics can be attributed to the deposition process and sintering temperature and conditions.

Overall, the density, permittivity, and $\mathrm{d}_{33}$ of the composite transducers tend to decrease as the percent of BiTi is increased in the samples, while the conductivity increases. Contrary to these trends, the signal amplitude, center frequency, and bandwidth seem to be relatively unaffected by the less than ideal dielectric properties of the fabricated transducers. From this small set of data it appears that if one of the composites fabricated through a spray-on process is going to be used as a transducer driven at low voltages then pure BiTi should be chosen; however, if the density, permittivity or conductivity is of significance then the user should consider adding PZT to the compound to increase these properties.

\section{REFERENCES}

[1] Sinding, Kyle M., Cliff Searfass, Nathan Malarich, Brian Reinhardt, and Bernhard Tittmann, "High Temperature Ultrasonic Transducers for the Generation of Guided Waves for Non-destructive Evaluation of Pipes." Review of Progrss in Quantitative Non-Destructive Evaluation,. AIP Conf.Proc., 302-307(2014)

[2] Searfass, C.T. "Fabrication and Testing of Piezoelectric Bismuth Titanate for use as a high Temperature Ultrasonic Transducer" Master's Thesis, 2008. Pennsylvania State University, University Park, PA.

[3] Kobayashi, M., Olding, T.R., Zou, L., Sayer, M., Jen, C.-K., Rehmen, A.U. "Piezoelectric Thick Film Ultrasonic Transducers Fabricated By A Spray Technique." IEEE Ultrasonics Symposium, 2000: 985-989.

[4] Pheil, C.E. "Fabrication and Testing of High Temperature Ultrasonic Transducers" Undergraduate Thesis, 2012. Pennsylvania State University, University Park, PA. 
[5] Bednarcyk, Brett A., and Steven M. Arnold. Micromechanics Analysis Code by Generalized Method of Cells. Computer software. Vers. 4.0. N.p., n.d. Web. 18 Jan. 2014.

[6] Torah, R. N., S. P. Beeby, and N. M. White. "Experimental Investigation into the Effect of Substrate Clamping on the Piezoelectric Behavior of Thick-film PZT Elements." Journal of Applied Physics 37 (2004): 1-5. Web. 16 Jan. 2014.
[7] Randall, Clive, Namchul Kim, John-Paul Kucera, Wenwe Cao, and Thomas Shrout. "Intrinsic and Extrinsic SizeEffects in Fine-Grained Mophotropic-Phase-Boundary Lead Zirconate Titanate Ceramics." Journal of American Ceramic Society 81 (1998): 677-88. Web. 18 Jan. 2014.

[8] Baun, Jim. "Transducers." Physical Principles of General and Vascular Sonography.N.p.: n.p., n.d. 107-14. Print. 